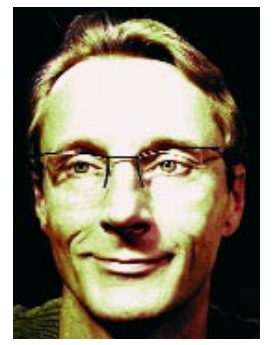

Jürgen Staedt

\title{
Schlafstörungen bei Demenzen
}

Jürgen Staedt

Klinik für Psychiatrie und Psychotherapie - Memory Clinic, Vivantes Klinikum Spandau, Berlin

psychoneuro 2004; 30 (9): 497-502

$\mathrm{M}$ it dem Alter treten sowohl qualitative als auch quantitative Veränderungen des Schlafes auf, und etwa 38\% der Älteren berichten gemäß epidemiologischen Untersuchungen über Schlafstörungen. Einschlafstörungen beklagen $36 \%$, und bis zu $29 \%$ der über 65-Jährigen berichten über Durchschlafstörungen. Neben somatischen Erkrankungen werden Schlafstörungen im Alter durch fehlende Tagesstruktur, Nickerchen (Napping) und nicht ausreichende körperliche Aktivität begünstigt.

Etwa 40\% der Alzheimer-Demenz-Patienten entwickeln eine Schlafstörung. Eine häufige Störung des Schlafablaufs bei dementen Patienten ist das Sundowning. Hierbei handelt es sich um eine in der Dämmerung oder frühen Nacht auftretende Verwirrtheit. Die Angaben in der Literatur zur Häufigkeit des Sundownings schwanken und liegen bei Heimpatienten in der Größenordnung von $10-25 \%$, sollen aber bei nicht in Heimen lebenden Alzheimer Patienten mit bis zu 66\% noch deutlich höher liegen. Mit zunehmender Schwere der Demenz kann sich die Störung des Schlaf-/ Wachrhythmus verstärken und in schweren Fällen auch zu einer völligen Desorganisation führen. Gerade die Störungen des Schlaf-/Wachrhythmus stellen Angehörige und behandelnde Ärzte vor große Probleme und sind der häufigste Grund für Heimeinweisungen (15).

Etwa 40\% der Demenzpatienten entwickeln eine Schlafstörung. Klinisch beginnt die Symptomatik mit vermehrten Aufwachvorgängen, die zunehmend in eine Fragmentation des Nachtschlafes münden können. Der Zerfall der zirkadianen Rhythmik und die zunehmenden kognitiven Defizite im Rahmen der Demenz stehen in Beziehung zu funktionellen und neuroanatomischen Veränderungen, die die Therapie komplizieren. Benzodiazepine können teilweise hilfreich sein, aber auch paradoxe Reaktionen hervorrufen. Neuroleptika sind in vielen Fällen unverzichtbar, weisen aber ein erhöhtes Risiko für zerebrovaskuläre Ereignisse auf. In diesem Zusammenhang sind nicht pharmakologische Optionen wie der Einsatz von Lichttherapie und körperlicher Aktivität zur Stabilisation des Schlaf-/Wachrhythmus von Interesse. Auch Cholinesterasehemmer und Melatonin können zu einer Stabilisierung des Schlaf-/Wachrhythmus beitragen.

\section{Pathophysiologische Grund- lagen der Veränderung des Schlaf-/Wachrhythmus}

Für die Therapie von Schlafstörungen bzw. Schlaf-/Wachrhythmusstörungen bei Demenzen ist es hilfreich, sich mit den pathophysiologischen Veränderungen des ZNS bei dementiellen Erkrankungen auseinander zu setzen. Da in etwa 70\% der dementiellen Erkrankungen eine Alzheimer Pathologie zugrunde liegt, werden im Folgenden die Auswirkungen der neuropathophysiologischen Veränderungen der Alzheimer Demenz auf den Schlaf-/WachRhythmus erörtert.

Mit dem Alter kommt es zu einer abnehmenden Konsolidierung des NONREM-Schlafes. Der NONREMSchlaf Stadium III und IV reduziert sich, und der leichte Schlaf Stadium I nimmt zu, gleichzeitig finden sich häufigere nächtliche Wachphasen und Wechsel der Schlafstadien (13).

Gerade die gestörte Konsolidierung des NONREM-Schlafes kann sich negativ auf kognitive Leistungen auswirken, da der NONREM-Schlaf auch mit der Konsolidierung des deklarativen Gedächtnisses und der Funktionsfähigkeit unseres Arbeitsgedächtnisses (im dorsolateralen präfrontalen Kortex) in Verbindung gebracht wird. Dies wird um so verständlicher, wenn wir uns vergegenwärtigen, dass Gedächtnisleistungen sich auf neuronaler Ebene in der Bahnungen bestimmter Verschaltungsmuster \& Rezeptorsensitivitäten und Rezeptordichten widerspiegeln (14).

Schlafstörungen haben auch einen Einfluss auf Gedächtnisprozesse. So zeigen neueste Daten, dass eine subjektiv erlebte Schlafstörung bei Älteren im 3-Jahres-Follow-up negativ mit der kognitiven Leistungsfähigkeit assoziiert war (5).

Neben den kognitiven Störungen stehen auch die Veränderungen des Schlafes bei der Alzheimer Demenz in Beziehung zu degenerativen Veränderungen des Nucleus basalis Meynert (NBM). Der NBM ist ein 
cholinerges, zum aufsteigenden aktivierenden retikulären System (ARAS) gehörendes Kerngebiet, welches den Neokortex versorgt. Dort senkt Azetylcholin das Ruhe-/spannungsabhängige Kalium-Membranpotential und steigert somit die neuronale Erregbarkeit (Reagibilität). Zusätzlich hemmen cholinerge Fasern des NBM auch in der Wachphase die Nuclei retikulares thalami. Während der Schlafphase fällt dieser hemmende Einfluss weg und es kommt über die Nuclei retikulares thalami zu GABAerg modulierten NONREM-Schlaf-Synchronisationen. So ist es verständlich, dass es bei der Alzheimer Demenz durch ein zunehmendes cholinerges Defizit zu EEG-Frequenzverlangsamungen kommt, die mit zunehmender Demenzausprägung eine Differenzierung des Schlaf-/Wach-EEG's erschweren. Entsprechend zeigt sich im Tierexperiment nach Läsionen des NBM eine diffuse EEG-Frequenzverlangsamung. In diesem Zusammenhang ist es interessant, dass die bei Patienten mit Lewy Körperchen Demenz häufig auftretende Fluktuation der Bewusstseinshelligkeit durch eine besonders ausgeprägte Degeneration der cholinergen Projektionen zu den Nuclei retikularis thalami erklärbar ist.

Die zirkadiane Regulation der Schlaf-/Wachperiodik flacht ebenfalls mit zunehmender Demenzschwere ab. Dazu passend beeinflussen cholinerge Bahnen des NBM auch die metabolische Aktivität des Nucleus suprachiasmaticus (NSC), sodass es im Tierexperiment nach Läsionen des NBM zu einer Reduktion der Nervenzellzahl/größe im NSC kommt. Die abnehmende Aktivität des NSC und die mit zunehmender Krankheitsprogression reduzierte Melatoninsynthese begünstigen zusammen mit verminderten externen Zeitgebern (körperliche Aktivität, soziale Isolation, geringe Lichtstärke in Wohnbereichen) die Störung des Schlaf-/Wachrhythmus im Demenzprozess.

Zusammenfassend lässt sich feststellen, dass die Schlaf-/Wachrhythmik maßgeblich durch cholinerge Neurone des NBM beeinflusst wird.

\section{Sundowning, Schlaf-/Wach- rhythmusstörung, REM- Schlafverhaltensstörung}

Sundowning findet sich bei Patienten mit Alzheimer-Demenz oder auch bei der Lewy Körperchen Demenz. Beim Sundowning handelt es sich typischerweise um eine in der Dämmerung oder abends auftretende Unruhe und Erregtheit, bei der die Patienten desorientiert sind, oft unkontrollierbar schreien, schwer zu beruhigen sind und sich durch ihr Verhalten gefährden. Auslöser für die Unruhezustände sind häufig Vokalisationen von Mitpatienten oder irritierende Geräusche, z.B. durch Reinigungspersonal oder Essenswagen. Sundowning wird durch die Ausprägung der Demenz wie auch durch räumliche Veränderungen begünstigt. Da das Auftreten des Sundownings an das Vorliegen einer Demenz gebunden ist und in der Regel mit einem phase delay (Temperaturmaximum verschoben) gegen Abend auftritt, handelt es sich pathophysiologisch wahrscheinlich um eine kortikale Aktivierung (Arousalreaktion) bei gleichzeitig reduzierter cholinerger „Basisstimulation“ des Kortex, wie sie oben beschrieben wurde. Zusätzlich wird in der Dämmerung noch die indirekte Licht-vermittelte Aktivierung reduziert. Vereinfacht könnte man auch sagen, es kommt beim Sundowning in der Dämmerung bei reduzierter NSC-Aktivierung zu einem Arousal bei „abgeschaltetem" - auf NONREM-Schlaf eingestellten - Neokortex. Die Folge ist, dass der zur Agitation führende Stimulus nicht bearbeitet werden kann und die Agitation bestehen bleibt oder sogar noch zunimmt.

Vom Sundowning abzugrenzen ist die REM-Schlaf-Verhaltensstörung. Die REM-Schlaf-Verhaltensstörung betrifft in der Regel nur Männer und tritt auch im Vorfeld einer dementiellen Erkrankung auf, sodass sie nicht an das Vorliegen einer Demenz gebunden ist. Über 50\% der idiopathischen REM-Schlaf-Verhaltensgestörten entwickelten in Verlaufsuntersuchungen ein Parkinsonsyndrom mit oder ohne Demenz. Sehr häufig tritt die REM-Schlafverhaltensstörung bei Lewy Körperchen Demenzen auf. Durch das Ausbleiben der motori- schen Hemmung in der REM-Schlafphase kommt es ein- bis zweimal pro Woche, teilweise bis zu viermal nachts, zum Ausagieren von bedrohlichen Trauminhalten (Flucht- und/ oder fremdaggressivem Verhalten), die auf Nachfrage im Gegensatz zum Sundowning auch erinnert werden können. Es handelt sich um eine motorische Störung, weil die im REMSchlaf normalerweise vorliegende Muskeltonuserniedrigung ausbleibt und so die geträumten Impulse „ausgelebt" werden. Ursächlich hierfür könnte die Degeneration von cholinergen pedunculopontinen Neuronen sein, die den Muskeltonus im Schlaf beeinflussen. In der Anamnese der Patienten findet sich häufig ein Sprechen im Schlaf.

\section{Therapie: Sundowning und Schlaf-/Wachrhythmus- störung \\ Schlafhygiene}

Da die Aktivitätsabnahme im SCN und NBM im Zusammenhang mit dem Auftreten des Sundownings und einer Schlaf-/Wachrhythmusstörung steht, sollte in erster Linie versucht werden, durch nicht-medikamentöse Therapiemaßnahmen die zirkadiane Rhythmik zu stabilisieren. Deshalb sollte man sich bei dementen Patienten mit Sundowning und Schlaf-/Wachrhythmusstörung zunächst einmal von den Bezugspersonen den Tagesablauf schildern lassen. Es sollte sichergestellt werden, dass die Betroffenen einen gleichmäßigen und stabilen Tagesrhythmus (Essen, Toilettengänge etc.) verbunden mit ausreichenden sozialen Kontakten haben und auch die körperliche Aktivierung nicht zu kurz kommt. Dazu passend konnte in Untersuchungen durch eine soziale Aktivierung einem polyphasischen Schlafrhythmus entgegengewirkt werden. Weiterhin ist eine klare Abgrenzung der Hell-Dunkel-Phasen zwingend notwendig. Im Heimbereich wird zum Teil im Median nur eine Lichtstärke von 54 Lux gemessen, und die Bewohner verbringen ca. nur zehn Minuten mit mehr als 1000 Lux (12). Im Vergleich dazu erreichen wir in unseren beleuchteten Arbeitsräumen 300-500 Lux. Selbst an be- 
wölkten Wintertagen im Freien beträgt die Lichtstärke noch 3 000-4 000 Lux. Der positive Effekt von Tageslicht auf den Schlaf konnte bei älteren Schlafgestörten nachgewiesen werden. In einer Untersuchung führte eine Tageslichexposition von 10:00-12:00 Uhr und von 14:00-16:00 Uhr zu erhöhten Melatoninspiegeln und einer Schlafverbesserung.

\section{Lichttherapie}

Bei dementen Patienten reduziert abendliche Lichttherapie nächtliche motorische Unruhe (4), aber auch morgendliche Lichttherapie ist wirksam (9). Auch eine indirekte Lichttherapie mit verstärkter Lichtintensität in den Aufenthaltsräumen führt zu einer Stabilisation des Schlaf-/Wachrhythmus (17). Neben der Stabilisation des Rhythmus konnte auch unter morgendlicher und abendlicher Lichttherapie eine Verbesserung der Kognition im Mini-Mental-State-Test nachgewiesen werden. Bei nicht ausreichender Lichtstärke tagsüber ist daher bei Schlaf-/Wachrhythmusstörungen eine 30-minütige Lichttherapie mit 10000 Lux empfehlenswert, die bezüglich der zeitlichen Belastung auch gut in den Stationsalltag integrierbar ist. Alternativ können auch 2500 Lux über zwei Stunden angewendet werden. Allerdings bleibt anzumerken, dass schwer demente Patienten mit ausgeprägterer Degeneration des NSC von der Lichttherapie nur (noch) begrenzt profitieren können. Sogar die Simulation von Sonnenauf- und -untergang wirkt sich positiv auf die Einschlaflatenz, nächtliche Unruhezustände und auch auf die Schlafdauer bei älteren Patienten mit fortgeschrittener Demenz aus. Um eine Umsetzung von schlafhygienischen Maßnahmen im Alltag zu gewährleisten, ist eine entsprechende Aufklärung der Angehörigen von Demenzpatienten notwendig. Erste Untersuchungen zeigen, dass dies zu einer deutlichen Stabilisierung des Schlaf-/WachRhythmus beiträgt

Erst, wenn diese Maßnahmen nicht zum erwünschten Erfolg führen, sollte an eine medikamentöse Behandlung gedacht werden.

\section{Melatonin}

Da Lichtexposition tagsüber zu erhöhten nächtlichen Melatoninspiegeln führt, ist auch der direkte Einsatz von Melatonin zur Behandlung von Schlafstörungen bei Dementen von Interesse. Zumal es bei der Alzheimer Demenz mit Progression der Erkrankung auch zu einer Abnahme des Melatonin-Liquorspiegel kommt. Melatonin soll in erster Linie über Mel1a-Rezeptoren die Aktivität des NSC hemmen und so indirekt durch Hemmung der zirkadianen „Wach-Aktivität“ den Schlaf begünstigen. Interessant in diesem Zusammenhang ist auch, dass Musiktherapie bei Alzheimer Patienten die Melatoninspiegel erhöht und möglicherweise über diesen Mechanismus auch beruhigend wirkt. Zwei plazebokontrollierte Studien zum Einsatz von Melatonin ergaben widersprüchliche Ergebnisse. Asayama et al. (1) fanden unter Melatonin (3 mg) nach vier Wochen eine signifikante Abnahme nächtlicher Aktivität, eine Verlängerung des Schlafes und eine Verbesserung der Werte im ADAS-Cog im Vergleich zu Plazebo, während Serfaty et al. (10) nach zwei Wochen unter $6 \mathrm{mg}$ Melatonin keine Verbesserung des Schlafes fanden. Die Wirksamkeit von Melatonin sollte daher erst nach längerfristiger Gabe überprüft werden. Dazu passend fanden Cardinali et al. (2) im VierMonatsverlauf unter $6 \mathrm{mg}$ Melatonin einen positiven Einfluss auf Sundowning und Schlaf (Melatonin kann über die internationale Apotheke bezogen werden, $3 \mathrm{mg}$ Melatonin kosten ca. 40 Cent/pro Tag).

\section{Therapie der REM-Schlaf- verhaltensstörung}

In der Regel kann die REMSchlafverhaltensstörung längerfristig erfolgreich ohne Gewöhnungseffekte mit Clonazepam in einer Dosierung von 0,5-1 mg behandelt werden. Auch eine Erhöhung der abendlich gegebenen Azetylcholinesterasehemmer-Dosis kann sich positiv über eine Verstärkung der cholinerg vermittelten Muskeltonuserniedrigung im REM-Schlaf auswirken. Bei ungenügendem Behandlungserfolg kann gegebenenfalls eine
Kombination mit Melatonin in einer Dosis zwischen 3-6 mg in Erwägung gezogen werden, allerdings liegen keine kontrollierten Studien vor. Abschließend sei noch erwähnt, dass serotonerg wirkende Antidepressiva zu einer Muskeltonuszunahme während des REM-Schlafes führen und somit zumindest theoretisch das Risiko der Entwicklung einer REMVerhaltensstörung begünstigen könnten. Differentialdiagnostisch ist bei akut auftretender REM-SchlafVerhaltensstörung auch an eine medikamentöse Intoxikation oder an ein Entzugssyndrom zu denken.

\section{Allgemeine Pharmako- therapie von Schlaf-/Wach- rhythmusstörungen} Azetylcholinesterasehemmer Nächtliche Unruhezustände im Rahmen von Schlaf-/Wachrhythmusstörungen bei Demenzen sind zum Teil schwierig von einer deliranten Symptomatik abzugrenzen. Die Abgrenzungsschwierigkeit liegt u.a. darin begründet, dass die cholinergen, zum aufsteigenden aktivierenden retikulären System (ARAS) gehörenden Bahnen, maßgeblich Arousal, Aufmerksamkeit, Gedächtnis und Schlaf-/Wachrhythmus beeinflussen. Folglich können sich Störungen des cholinergen Systems je nach Vulnerabilität und Ausprägung entlang eines Kontinuums unterschiedlich auf höhere kortikale Funktionen (Bewusstsein, Aufmerksamkeit) und/ oder Schlaf-/Wachrhythmus auswirken. Bezugnehmend auf diese Überlegungen ist auch verstehbar, dass z.B. demente Patienten häufig im Rahmen fieberhafter Infekte eine delirante Symptomatik entwickeln. Denn dabei kommt es zur Bildung von endogenen anticholinergen Substanzen (3), die durch eine Blockade von Muskarinrezeptoren das schon bestehende cholinerge Defizit so verstärken könnten, sodass die cholinerge neokortikale Aktivierung nicht ausreicht und sich ein reversibles Delir mit Einschränkung der höheren kortikalen Funktionen entwickeln kann.

Deshalb ist es wichtig, grundsätzlich bei der Verordnung von Azetylcholinesterasehemmern nicht nur an die Kognition, sondern auch 


\section{Tab. 1 Wirkdauer und Eliminationshalbwertszeiten einiger Benzodiazepine u.a. Hypnotika}

\begin{tabular}{|c|c|c|c|c|}
\hline $\begin{array}{l}\text { Chemische } \\
\text { Bezeichnung }\end{array}$ & $\begin{array}{l}\text { Präparate- } \\
\text { namen }^{\circledR}\end{array}$ & $\begin{array}{l}\mathbf{t}^{1} / 2(\mathrm{~h}) \\
\text { Metabolit }^{*}\end{array}$ & $\begin{array}{l}\text { Verlängerung } \\
\mathrm{t} 1 / \mathrm{h}(\mathrm{h}) \\
\text { im Alter (\%) }\end{array}$ & $\begin{array}{l}\text { Äquivalenz } \\
\text { zu } 10 \mathrm{mg} \\
\text { Diazepam** }\end{array}$ \\
\hline Alprazolam & Tafil $^{\circledR}$ & $10-15$ & $40 \%$ & $1,5 \mathrm{mg}$ \\
\hline Bromazepam & Lexotanil $^{\circledR}$ & $12-28$ & $75 \%$ & $6 \mathrm{mg}$ \\
\hline Brotizolam & Lendormin ${ }^{\circledR}$ & $4-7$ & $95 \%$ & $0,5 \mathrm{mg}$ \\
\hline Chlordiazepoxid & Librium $^{\circledR}$ & $10-90^{*}$ & $80-350 \%$ & $20 \mathrm{mg}$ \\
\hline Clobazepam & Frisium ${ }^{\circledR}$ & $10-120^{*}$ & $60-180 \%$ & $20 \mathrm{mg}$ \\
\hline Clonazepam & Rivotril ${ }^{\circledR}$ & $24-40$ & - & $2 \mathrm{mg}$ \\
\hline Clorazepat & Tranxillium ${ }^{\circledR}$ & $1-82^{*}$ & $90-195 \%$ & $20 \mathrm{mg}$ \\
\hline Clotiazepam & Trecalmo ${ }^{\circledR}$ & $3-15$ & $20 \%$ & $5 \mathrm{mg}$ \\
\hline Diazepam & Valium $^{\circledR}$ & $30-80^{*}$ & $125-200 \%$ & $10 \mathrm{mg}$ \\
\hline Flunitrazepam & Rohypno|® ${ }^{\circledR}$ & 18 & $50 \%$ & $1 \mathrm{mg}$ \\
\hline Flurazepam & Dalmadorm $^{\circledR}$ & $2-100^{*}$ & $35-115 \%$ & $30 \mathrm{mg}$ \\
\hline Loprazolam & Sonin ${ }^{\circledR}$ & $9^{*}$ & $120 \%$ & $1,5 \mathrm{mg}$ \\
\hline Lorazepam & Tavor $^{\circledR}$ & $10-14$ & - & $2 \mathrm{mg}$ \\
\hline Lormetazepam & Noctamid $^{\circledR}$ & $10-14$ & - & $1 \mathrm{mg}$ \\
\hline Midazolam & Dormicum $^{\circledR}$ & $1-3$ & $20-25 \%$ & $7,5 \mathrm{mg}$ \\
\hline Nitrazepam & Mogadan ${ }^{\circledR}$ & $18-30$ & $40 \%$ & $5 \mathrm{mg}$ \\
\hline Nordazepam & Tranxillium ${ }^{\circledR} \mathrm{N}$ & $50-98$ & $90-195 \%$ & $20 \mathrm{mg}$ \\
\hline Oxazepam & Adumbran ${ }^{\circledR}$ & $5-15$ & - & $40 \mathrm{mg}$ \\
\hline Prazepam & Demetrin $^{\circledR}$ & $50-90^{*}$ & $90-195 \%$ & $20 \mathrm{mg}$ \\
\hline Temazepam & Remestan $^{\circledR}$ & $5-13$ & - & $20 \mathrm{mg}$ \\
\hline Triazolam & Halcion ${ }^{\circledR}$ & $2-4^{*}$ & - & $0,5 \mathrm{mg}$ \\
\hline Zaleplon & Sonata ${ }^{\circledR}$ & 1 & $\approx 50 \%$ & $30 \mathrm{mg} ?$ \\
\hline Zolpiden & Stilnox ${ }^{\circledR}$ & $0,7-3,5$ & $15 \%$ & $10 \mathrm{mg}$ \\
\hline Zopiclon & Ximovan $^{\circledR}$ & $5^{*}$ & $60-80 \%$ & $7,5 \mathrm{mg}$ \\
\hline
\end{tabular}

Rot unterlegt sind einige nach unserer Erfahrung aufgrund ihrer Halbwertszeit bzw. fehlenden Verlängerung der Halbwertszeit im Alter zur Therapie geeignete Benzodiazepine bzw. NichtBenzodiazepine, Clonazepam ist trotz langer Halbwertszeit aufgeführt, da es als Mittel erster Wahl zur Therapie der REM-Schlafverhaltensstörung zu sehen ist.

t11/2 (h): Halbwertszeit; * : aktive Metaboliten; ${ }^{* *}$ : Erfahrungswerte bzw. Schätzungen ohne Gewähr ning einsetzbar (2 bis 4 Kps. zeitlich versetzt), da es aufgrund der kurzen Halbwertszeit (3-6 h) gut steuerbar ist. Eingeschränkt wird die Anwendungsmöglichkeit allerdings dadurch, dass Clomethiazol wegen der atemdepressiven Wirkung nicht bei respiratorischer Insuffizienz eingesetzt werden darf. Generell sollte bei der Verordnung von Benzodiazepinen auch an das Vorliegen eines Schlafapnoesyndroms gedacht, da Benzodiazepine durch Muskelrelaxation und Arousalschwellenerhöhung die Sauerstoffentsättigung verstärken können.

\section{Neuroleptika}

Problematisch erscheint der Einsatz von Neuroleptika, da sowohl die Fa. Janssen-Cilag als auch Lilly Deutschland Anfang März 2004 in Rote Hand Briefen über ein erhöhtes Risiko zerebrovaskulärer Ereignisse bei Demenzpatienten unter der Therapie mit Risperidon bzw. Olanzapin berichtet haben (siehe auch 6). Olanzapin erhielt daraufhin in der Fachinformation den Warnhinweis: „Olanzapin ist für die Behandlung von Psychosen und/oder Verhaltensstörungen im Zusammenhang mit einer Demenz nicht zugelassen und die Anwendung in dieser speziellen Patientengruppe wird nicht empfohlen, da die Mortalität und das Risiko eines zerebrovaskulären Zwischenfalls erhöht ist." Bei Risperidon wurde die Indikation enger gefasst, statt in der Indikation „Chronische Aggressivität" wurde die Zulassung begrenzt auf: „Schwere chronische Aggressivität, durch die sich die Patienten selbst und andere gefährden, oder psychotische Symptome bei Demenz, durch die die Patienten erheblich beeinträchtigt werden." Die Einschränkung der Zulassung für Risperidon und Olanzapin grenzt die therapeutischen Optionen stark ein, obwohl es bisher nur für Risperidon und Olanzapin eine ausreichend große Anzahl von randomisierten, doppelblinden Studien gibt, die eine realistische Bewertung des Nutzen/Risikoprofils der Substanzen bei Demenzpatienten erlauben. Mit Blick auf diese Anwendungseinschränkungen dürften Neuroleptika folglich nicht mehr zur Therapie von 
Schlaf-/Wachrhythmusstörungen eingesetzt werden und wir müssten verstärkt Benzodiazepine verordnen. In diesem Zusammenhang ist die letztes Jahr von Kozma et al. (7) als Poster auf dem International College of Geriatric Psychoneuropharmacology in Puerto Rico präsentierte Studie von Interesse. In der Studie wurden im Zeitraum 1999-2001 die Verordnung Azetylcholinesterasehemmern, Olanzapin, Risperidon, Haloperidol und Benzodiazepinen bei insgesamt 14648 Patienten (> 60 Jahre mit Demenz; Ausschluss vaskuläre Demenz) erfasst und das Auftreten eines zerebrovaskulären Ereignisses innerhalb von 90 Tagen nach Therapiebeginn dokumentiert. Interessanterweise zeigte sich dort, dass nicht nur unter Risperidon und Olanzapin, sondern auch unter Benzodiazepinen und Haloperidol das relative Risiko für zerebrovaskuläre Ereignisse erhöht ist (Abb. 1). Somit muss zum gegenwärtigen Zeitpunkt davon ausgegangen werden, das auch typische Neuroleptika und Benzodiapine ein erhöhtes Risiko für zerebrovaskuläre Ereignisse aufweisen.

Aus diesem Grunde sollte der Einsatz von Neuroleptika und Benzodiazepinen erst nach Ausschöpfung der oben erwähnten schlafhygienischen Maßnahmen und Beginn einer Therapie mit Cholinesterasehemmern erfolgen.

Als Mittel der ersten Wahl ist dann Risperidon (0,5-0-0,5 mg) anzusehen. Risperidon führte in Untersuchungen bei dementen Patienten zur Stabilisierung des Schlaf-/Wachrhythmus und zeigte auch im Vergleich zu Melperon weniger Nebenwirkungen wie Schwindel, Gangunsicherheit und Tagessedierung, ohne dass sich beide Substanzen in ihrem Einfluss auf den Nachtschlaf unterschieden. Als Mittel zweiter Wahl sollten bei unzureichendem Therapieerfolg unter Risperidon Melperon oder Dipiperon zum Einsatz kommen. Haloperidol sollte nicht zur Therapie von Schlafstörungen eingesetzt werden, da Haloperidol gemäß Cochrane Review (8) nicht für die Therapie von Unruhezuständen bei Demenzpatienten empfohlen werden kann und es auch Hinweise da-

\section{Abb. 1 Odds Ratio (relatives Risiko) für zerebrovaskuläre Ereignisse, Referenzsubstanz Azetylcholinesterase- hemmer}

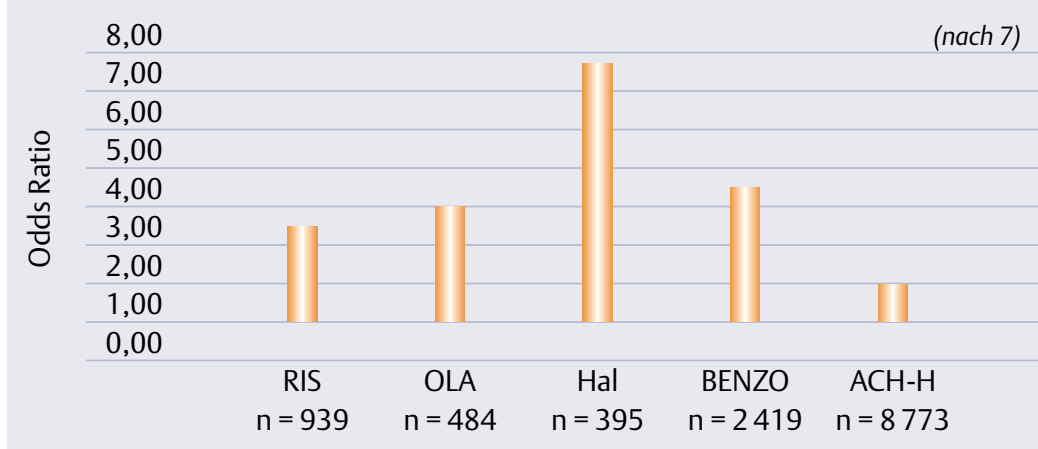

Die Odds Ratio beschreibt ein Verhältnis, eine Odds Ratio von 1 bedeutet „kein Unterschied“. Ein Wert grösser als 1 beschreibt ein Risiko für ein Ereignis, ein Wert kleiner als 1 beschreibt einen „Schutz“ vor einem Ereignis. Der Wert für ACH-H ist gleich $1 \mathrm{gesetzt}$, da sich unter ACH-H bisher kein erhöhtes Risiko für zerebrovaskuläre Ereignisse gezeigt hat. Eine Odd Ratio von 3 bedeutet, das die Wahrscheinlichkeit für das Auftreten eines zerebrovaskulären Ereignisses gegenüber der Referenzsubsubstanz $(\mathrm{ACH}-\mathrm{H})$ um den Faktor 3 erhöht ist.

Abkürzungen: Ris = Risperidon; OLA = Olanzapin; Hal = Haloperidol; Benzo =

Benzodiazepine; $\mathrm{ACH}-\mathrm{H}=$ Azetylcholinesterasehemmer; $\mathrm{n}$ = Anzahl der Patienten

rauf gibt, dass Haloperidol die zirkadiane Rhythmik negativ beeinflussen kann. Alternativ kann hier auch an den Einsatz des gut sedierend wirkenden Quetiapins gedacht werden (0-0-25mg; langsame Steigerung auf bis zu $0-0-100 \mathrm{mg}$ ). Quetiapin ist aufgrund der kurzen Halbwertszeit $(\approx 7 \mathrm{~h})$ gut steuerbar, hat keine anticholinergen Nebenwirkungen und ein geringes Risiko hinsichtlich extrapyramidalmotorischer Nebenwirkungen. Es ist allerdings für diese Indikation noch nicht in kontrollierten Studien untersucht.

\section{Zusammenfassung}

Schlaf-/Wachrhythmusstörungen bei dementen Patienten stehen in Beziehung zu Aktivitätsänderungen im NSC und NBM. Die Therapiemaßnahmen sollten deshalb in erster Linie darauf abzielen, das zirkadiane System zu stimulieren. Bei Patienten mit Schlafstörungen bzw. Schlaf-/Wachrhythmusstörungen sollte genau die Struktur des Tagesablaufes erfasst werden, um durch die Sicherstellung eines stabilen Tagesrhythmus mit einer ausreichenden körperlichen Aktivierung (mit Aufenthalt im Freien und einer Begrenzung von „Nickerchen“) physiologisch den Ruhe-Aktivitäts- rhythmus zu verbessern. Bei nicht ausreichender Lichtstärke im Wohnbereich sollte tägliche Lichttherapie zu Einsatz kommen.

Um auch eine Umsetzung dieser schlafhygienischen Maßnahmen im Alltag zu gewährleisten, ist eine entsprechende Aufklärung der Angehörigen von Demenzpatienten notwendig. Erste eigene Untersuchungen zeigen, dass die Information der Angehörigen zu einer deutlichen Stabilisierung des Schlaf-/ Wachrhythmus beiträgt.

Erst, wenn diese Maßnahmen nicht zum erwünschten Erfolg führen, sollte an eine medikamentöse Behandlung gedacht werden. Bei der Auswahl der Medikation sollten möglichst wenig sedierende Psychopharmaka zum Einsatz kommen, um nicht durch einen Überhang mit Sedierung am Vormittag den ohnehin reduzierten neuronalen Metabolismus (neuronale Aktivität) der Demenzpatienten noch zusätzlich zu schwächen. Langfristig kann hier eine fortgesetzte Sedierung der neuronalen Inaktivierung/Atrophie im Rahmen der Demenz Vorschub leisten und die nutzungsabhängige dynamische Plastizität neuronaler Verschaltungen schwächen. Daher sollte 


\section{Die verlorene Kunst des} Heilens

Lown, Bernhard: Die verlorene Kunst des Heilens. Anstiftung zum Umdenken. 2. Auflage. Schattauer Verlag, 2004. 307 Seiten, geb. € 34,95. ISBN: 3-79452347-4

etzt liegt die zweite bebilderte Auflage des Buches vor. Der Autor hat neue Kapitel über die Bedeutung von Plazebos und über den Boom von alternativen Heilmethoden ergänzt. Der Autor ist ein begnadeter Erzähler, humorbegabt, sodass auch die zweite Auflage denen zu empfehlen ist, die quasi aus Versehen die 1. Auflage nicht gelesen haben.

Prof. Dr. F. Reimer, Weinsberg

\section{Restless Legs: Wirksame Hilfe bei unruhigen Beinen}

Jörn P. Sieb: Restless Legs. Wirksame Hilfe bei unruhigen Beinen. TRIAS Verlag in MVS Medizinverlage Stuttgart, 2003. 128 S. Kartoniert. € 14,95. ISBN 3-83043099-X

$\mathrm{R}^{\mathrm{s}}$ estless Legs - ruhelose Beine: Das ist eine Diagnose, die eher harmlos klingt. Und doch würde sich ein betroffener Patient „am liebsten die Beine amputieren“, wenn die nächtlichen Missempfindungen ihn heimsuchen und hartnäckig am Einschlafen hindern. Diese drastische Aussage aus einem der zahlreichen Patientenbeispiele im Buch macht deutlich, wie quälend das Restless-LegsSyndrom (RLS) sein kann. Immerhin sind schätzungsweise 1,6 bis 8 Millionen Deutsche von dieser neurologischen Störung betroffen; bei vielen jedoch bleibt die Erkrankung unerkannt und dementsprechend auch unbehandelt.

Der Ratgeber von PD Dr. med. Jörn P. Sieb, der bis letztes Jahr die „Ambulanz für Bewegungsstörungen“ am Max-Planck-Institut für Psychiatrie in München-Schwabing leitete, informiert auf 128 Seiten wann und wie das RLS auftritt, welche möglichen Ursachen es gibt, wie die Diagnostik erfolgt und welche Selbsthilfe bei leichten Verlaufsformen bzw. Pharmakotherapie bei schweren Erkrankungen hilfreich ist.

Sicherlich hatte der Autor beim Verfassen des Werkes nicht nur seine Patienten, sondern auch ärztliche Kollegen als potenzielle Leser im Blick, denn obwohl das Buch patientengerecht geschrieben ist, enthält es doch alle nötigen Informationen, um auch dem Arzt einen guten Einstieg in die Thematik zu bieten. Es ist daher sowohl für Betroffene als auch für Ärzte, die mit der RLS-Diagnostik und -Therapie noch nicht vertraut sind, empfehlenswert. auch bei Schlaf-/Wachrhythmusstörungen an den Einsatz von Cholinesterasehemmern gedacht werden, da das cholinerge Systems maßgeblich an der Aufrechterhaltung der Vigilanz und der Regulation des Schlaf-/Wachrhythmus beteiligt ist.

\section{Sleep disorders in dementia}

Approximately 40\% of dementia patients develop a sleep disorder. Clinically the symptoms commence with wakening processes growing in frequency, which may finally result in a fragmentation of nocturnal sleep. The disintegration of the circadian rhythms and the increasing cognitive deficits within the scope of dementia are related to functional and neuroanatomical changes which complicate therapy. Benzodiazepine receptor agonists may be partly helpful, but may also provoke paradoxical reactions. Neuroleptics are indispensable in many cases, but exhibit a higher risk for cerebrovascular events. In this context non-pharmacological options such as the application of light therapy and physical activity are of particular interest in stabilizing the sleep-wake cycle. In addition to that, melatonin and cholinesterase inhibitors might also contribute to a stabilization of the sleep-wake cycle.

\section{Keywords}

sleep - sundowning - dementia RBD - melatonine - light treatment

\section{Literatur}

1. Asayama $\mathrm{K}$, Yamadera $\mathrm{H}$, Ito $\mathrm{T}$ et al. Double blind study of melatonin effects on the sleep-wake rhythm, cognitive and noncognitive functions in Alzheimer type dementia. J Nippon Med Sch 2003; 70: 334-341

2. Cardinali DP, Brusco LI, Liberczuk $C$ et al. The use of melatonin in Alzheimer's disease. Neuroendocrinol Lett 2002; 23 : 20-23

3. Flacker JM, Wei JY. Endogenous anticholinergic substances may exist during acute illness in elderly medical patients. J Gerontol. MS 2001; 56A: M353-M355

4. Haffmans PM, Sival RC, Lucius SA et al. Bright light therapy and melatonin in motor restless behaviour in dementia: a placebocontrolled study. Int J Geriatr Psychiatry 2001; 16: 106-110

5. Jelicic M, Bosma H, Ponds RW et al. Subjective sleep problems in later life as predictors of cognitive decline. Report from the Maastricht Ageing Study (MAAS). Int J Geriatr Psychiatry 2002; 17: 73-77
6. Jüptner M, Gastpar M. Todesfälle unter Risperidon und Olanzapin - was nun? Behandlung psychotischer Symptome bei Demenzpatienten. psychoneuro 2004; 30 (6): 314-216

7. Kozma CM, Engelhart LM, Long S, Greenspan A, Mahmoud R, Baser O. Absence of increased relative stroke risk in elderly dementia patients treated with risperidone versus other antipsychotics. ICGP Programm book, www.icgp.org/ICGP_2003_programm_boo k.pdf73,2003

8. Lonergan E, Luxenberg J, Colford J. Haloperidol for agitation in dementia. Cochrane Database Syst Rev 2002; 2: CD002852

9. Lyketsos CG, Lindell Veiel L, Baker A et al. A randomized, controlled trail of bright light therapy for agitated behaviors in dementia patients residing in long-term care. In J Geriatr Psychiatry 1999; 14: 520-525

10. Serfaty M, Kennell-Webb S, Warner J et al. Double blind randomised placebo controlled trial of low dose melatonin for sleep disorders in dementia. Int J Geriatr Psychiatry 2002; 17: 1120-1127

11. Shaw SH, Curson H, Coquelin JP. A double-blind, comparative study of zolpidem and placebo in the treatment of insomnia in elderly psychiatric in-patients. J Int Med Res. 1992; 20: 150-161

12. Shochat T, Martin J, Marler M et al. Illumination levels in nursing home patients: effects on sleep and activity rhythms. J Sleep Res 2000; 9: 373-337

13. Staedt J. Schlaf/zirkadiane Rhythmik bei Alzheimer Demenz. In: Calabrese P, Förstl H (Hrsg.). Psychopathologie und Neuropsychologie der Demenz. Lengerich: Pabst, 2000: 104-116

14. Staedt J, Stoppe G. Are sleep and ist disorders of interest for psychiatric and psychosomatic medicine? In: Diefenbacher A (Hrsg.). Advances in Psychosomatic Medicine. Basel: Karger, 2004, Vol 26: 1-6

15. Stoppe G, Brandt C, Staedt J. Behavioural problems associated with dementia: the role of newer antipsychiotics. Drugs and Aging 1999; 14: 41-54

16. Trinh NH, Hoblyn J, Mohanty S et al. Efficacy of cholinesterase inhibitors in the treatment of neuropsychiatric symptoms and functional impairment in Alzheimer disease: a meta-analysis.JAMA 2003; 289: 210-216

17. Van Someren EJ, Kessler A, Mirmiran M et al. Indirect bright light improves circadian rest-activity rhythm disturbances in demented patients. Biol Psychiatry 1997; 41: 955-963

18. Wynn Z], Cummings JL. Cholinesterase inhibitor therapies and neuropsychiatric manifestations of Alzheimer's disease. Dement Geriatr Cogn Disord 2004; 17: 100-108

\section{Korrespondenzadresse:}

Prof. Dr. med. Jürgen Staedt

Klinik für Psychiatrie und Psychotherapie Memory Clinic

Vivantes Klinikum Spandau

Griesingerstr. 27-33

10623 Berlin

E-Mail: juergen.staedt@vivantes.de 